\title{
Reconstrucción en amputaciones distales de dedos en niños con injerto compuesto y vendaje semioclusivo
}

\section{Reconstruction of Distal Finger Amputations in Children with Composite Autograft and Semi-Oclusive Dressing}

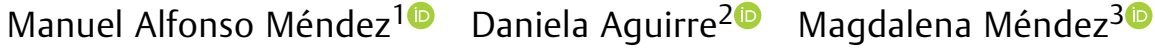 \\ ${ }^{1}$ Departamento de Ortopedia y Traumatología, Pontificia Universidad \\ Católica de Chile, Santiago, Chile \\ 2 Departamento de Ortopedia y Traumatología, Clínica Las Condes, \\ Región Metropolitana, Chile \\ Address for correspondence Manuel Alfonso Mendez, MD, Pontificia \\ Universidad Católica de Chile, Diagonal Paraguay 362, Edificio \\ Académico, piso 3. Santiago, Chile \\ (e-mail: mendezbecerram64@gmail.com).
}

3 Escuela de Medicina, Pontificia Universidad Católica de Chile,

Santiago, Chile

Rev Chil Ortop Traumatol 2021;62(2):e93-e98.

\section{Resumen \\ Palabras Clave \\ - amputación distal de dedos \\ - niños \\ - reconstrucción}

La reconstrucción de una amputación distal de dedo en un niño es un desafío. Los procedimientos propuestos son muchos, y los resultados no han sido buenos. La reconstrucción con reposición del segmento a modo de injerto compuesto, o con técnicas microquirúrgicas, parece ofrecer la mejor de las posibilidades, pues se conservan estructuras irremplazables, como el lecho ungueal y el hiponiquio, lo que permite que los niños mantengan un pulpejo anatómico y con función normal. Presentamos una serie de tres pacientes pediátricos tratados con una nueva técnica, que combina la reposición del segmento, como un injerto compuesto, y el uso de curación semioclusiva (composite autograft and semi-oclussive dressing, CASOD, en inglés). Hemos observado buenos resultados.

The reconstruction of finger tip amputation in children is challenging. There are many procedures described to treat this injury, none of which present optimal results. Repositioning of the amputated segment as an autograft or with microsurgical techniques seems to offer the best outcome. It enables the preservation of otherwise irreplaceable structures, such as the nail bed and the hyponychium, thus enabling children to mantain an anatomically and functionally normal finger pad. We present a series of three pediatric patients treated with tha new technique, which combines composite autograft and semi-oclusive dressing (CASOD). The results observed so far have been promising. recibido

27 de mes de julio de 2020

aceptado

02 de junio de 2020
DOI https://doi.org/

$10.1055 / \mathrm{s}-0041-1735292$. ISSN 0716-4548.
(C) 2021. Sociedad Chilena de Ortopedia y Traumatologia. All rights reserved.

This is an open access article published by Thieme under the terms of the Creative Commons Attribution-NonDerivative-NonCommercial-License, permitting copying and reproduction so long as the original work is given appropriate credit. Contents may not be used for commercial purposes, or adapted, remixed, transformed or built upon. (https://creativecommons.org/ licenses/by-nc-nd/4.0/)

Thieme Revinter Publicações Ltda., Rua do Matoso 170, Rio de Janeiro, RJ, CEP 20270-135, Brazil 


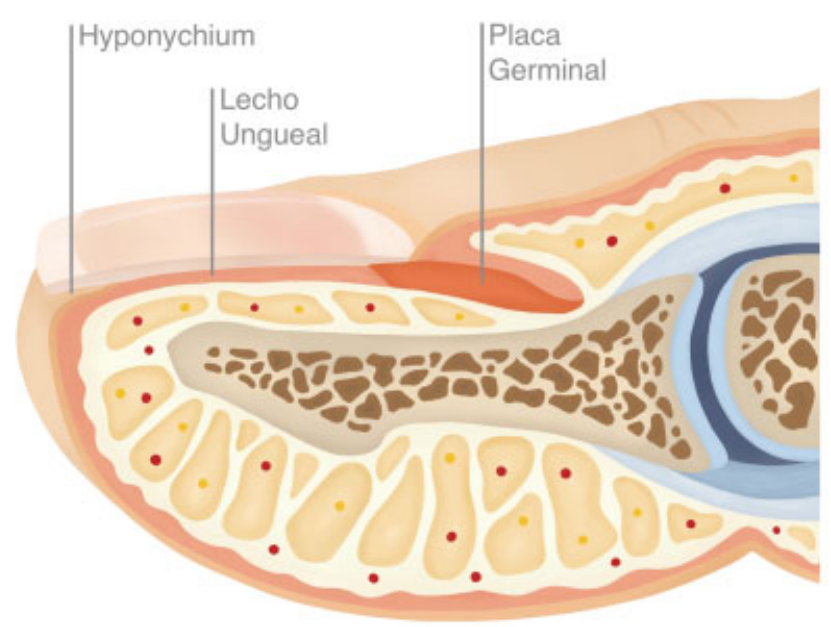

Fig. 1 Esquema del hiponiquio y del lecho ungeal.

\section{Introducción}

La amputación distal de dedos es la que ocurre distal a la inserción del tendón flexor profundo, y constituye una situación difícil de tratar en la población infantil. También es cierto que esto constituye principalmente un problema estético, y es altamente probable que no se traduzca en problemas funcionales de la mano.

El mecanismo de lesión casi siempre se asocia a un atrapamiento en una puerta.

Frente a una amputación distal de un dedo, muchas han sido las alternativas de tratamiento descritas para la población en general. Se ha planteado el manejo con un vendaje semioclusivo, ${ }^{1}$ el manejo de reposición a modo de injerto compuesto, ${ }^{2}$ la reconstrucción con un injerto de aparato ungueal más un colgajo de pulpejo, ${ }^{3}$ y el reimplante con uso de técnicas microquirúrgicas. ${ }^{4}$ Con todo, en general, los resultados en la población pediátrica siguen siendo no satisfactorios.

En el caso de hacer reimplantes con técnicas microquirúrgicas, las condiciones no son favorables por lo reducido de los diámetros de los vasos, y se requiere de un gran entrenamiento. El intento de hacer sólo la reposición de segmentos amputados a modo de injerto compuesto frecuentemente deja deformidades en la punta de los dedos. ${ }^{5}$ En el caso de los vendajes semioclusivos como técnica aislada, nunca logra regenerar el hiponiquio.

Postulamos que, en la reconstrucción de estas amputaciones, uno de los pasos críticos es conservar el lecho ungueal y el hiponiquio ( $\mathbf{- F i g . ~ 1 ) , ~ q u e ~ s o n ~ t e j i d o s ~}$ irremplazables. Es cierto que el largo del pulpejo y el aspecto estético del dedo los podemos conseguir con un colgajo de avance o axial. Pero es la conservación del lecho ungueal y del hiponiquio la clave para preservar la anatomía de la punta, evitando la deformidad en pico de loro, permitiendo así una adecuada pinza, y podemos tambien reducir la presencia de heridas e infecciones.

Así, el objetivo del tratamiento de estas lesiones debe ser conservar la longitud del dedo, dar un pulpejo blando, no doloroso, con un aspecto cosmético adecuado, y permitir una adecuada flexión distal del dedo.

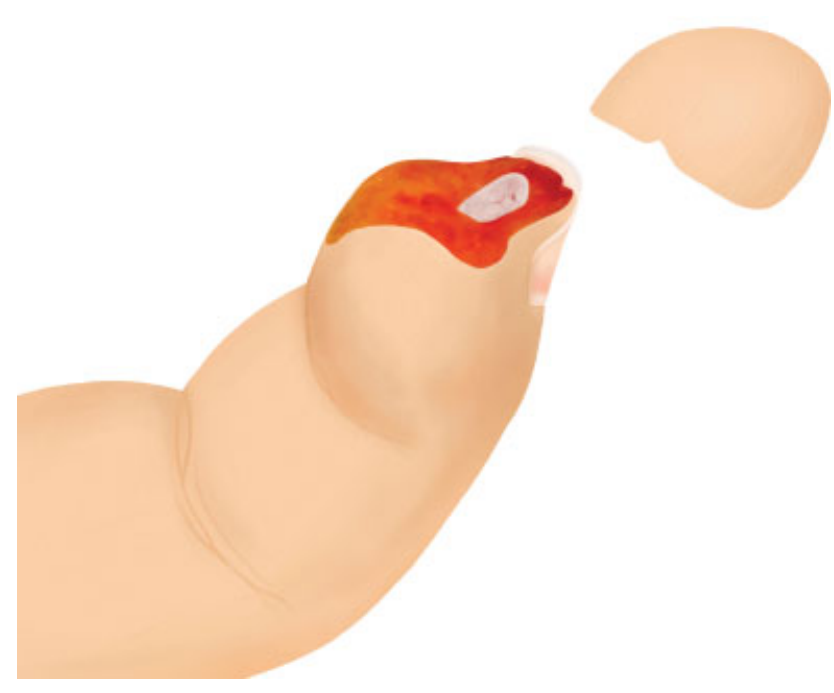

Fig. 2 Esquema de una amputación de pulpejo.

Tomando las diferentes propuestas observadas en la historia de estas lesiones, queremos presentar una nueva alternativa de tratamiento de las amputaciones distales de los dedos en población pediátrica, realizando la reconstrucción con reposición del segmento, como un injerto compuesto, combinándolo con vendaje semioclusivo.

\section{Procedimiento}

En pabellón, bajo sedación y anestesia troncular, se realiza un aseo del dedo lesionado y del fragmento amputado con solución fisiológica y clorhexidina; si hay componente óseo, este no se retira (-Fig. 2).

Se realiza una resección parcial del tejido graso del pulpejo amputado para disminuir el grosor del tejido que será repuesto (-Fig. 3). En el caso de que exista un componente óseo en la parte amputada, se fija con una aguja hipodérmica y finalmente se colocan puntos de piel a modo de afronte (-Fig. 4). Luego, se coloca un apósito de

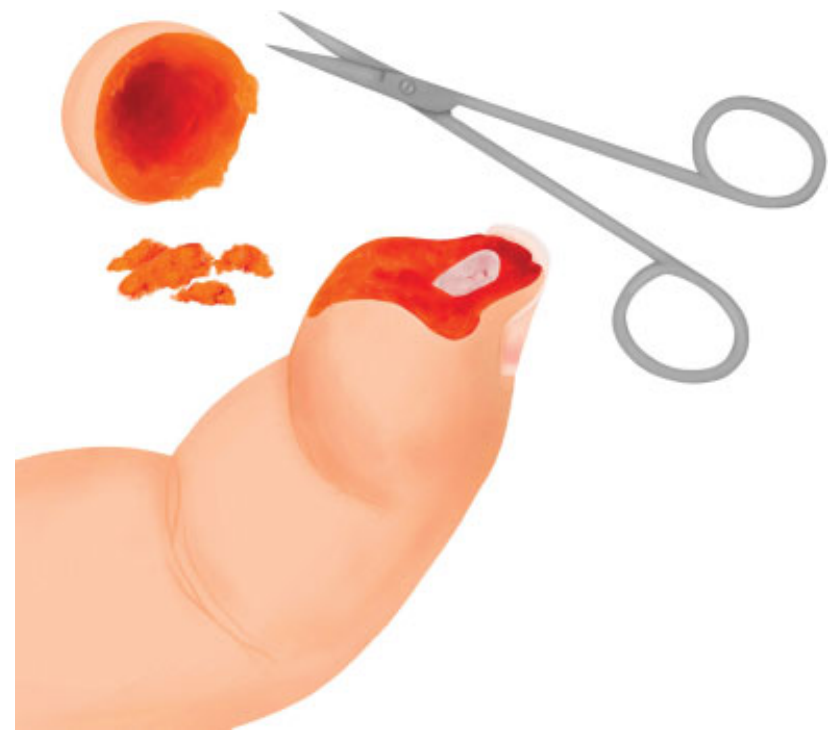

Fig. 3 Retiro de parte de tejido graso de pulpejo. 




Fig. 4 Injerto compuesto suturado.

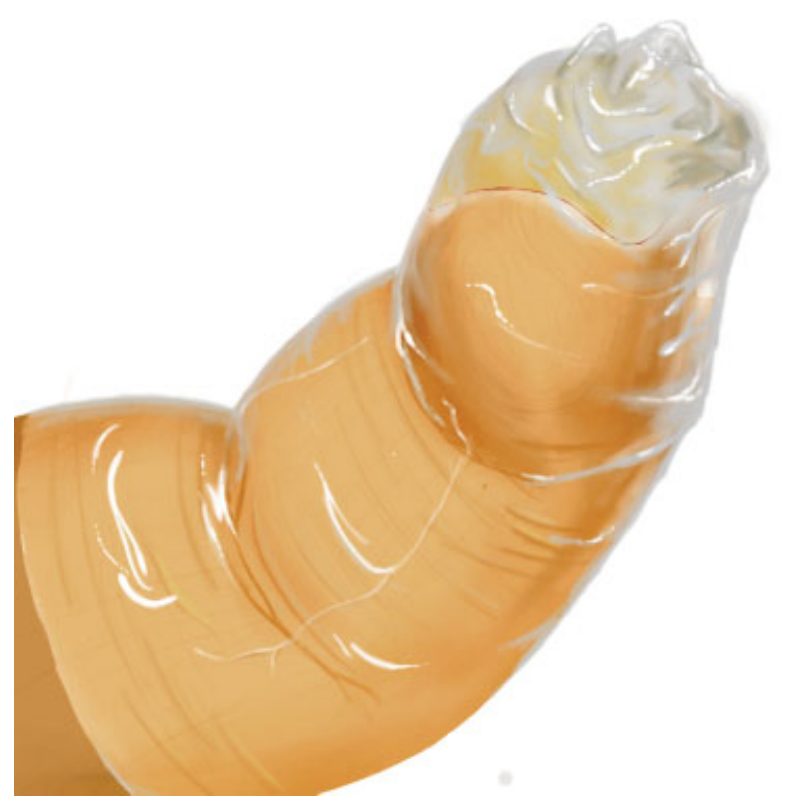

Fig. 5 Colocación de película transparente.

película transparente (Tegaderm Film, 3M, Saint Paul, MN, EEUU) (-Fig. 5), cuidando en sellar completamente el dedo para impedir la salida de líquidos. Luego de esto, se debe cuidar que el vendaje de película transparente permita observar que el capilar del dedo se llene adecuadamente. El sello de apósito de película se retira a las seis semanas. Se debe citar al paciente una vez por semana para verificar el correcto selle del dedo, la eventual presencia de infecciones, y advertir a los padres sobre el mal olor que puede derivar del tratamiento. Durante las seis semanas, el apósito de película no se retira, solo se refuerza.

\section{Caso 1}

Niña de 18 meses, con atrapamiento de dedo anular derecho en una bisagra de un juguete, que sufrió una amputación de la

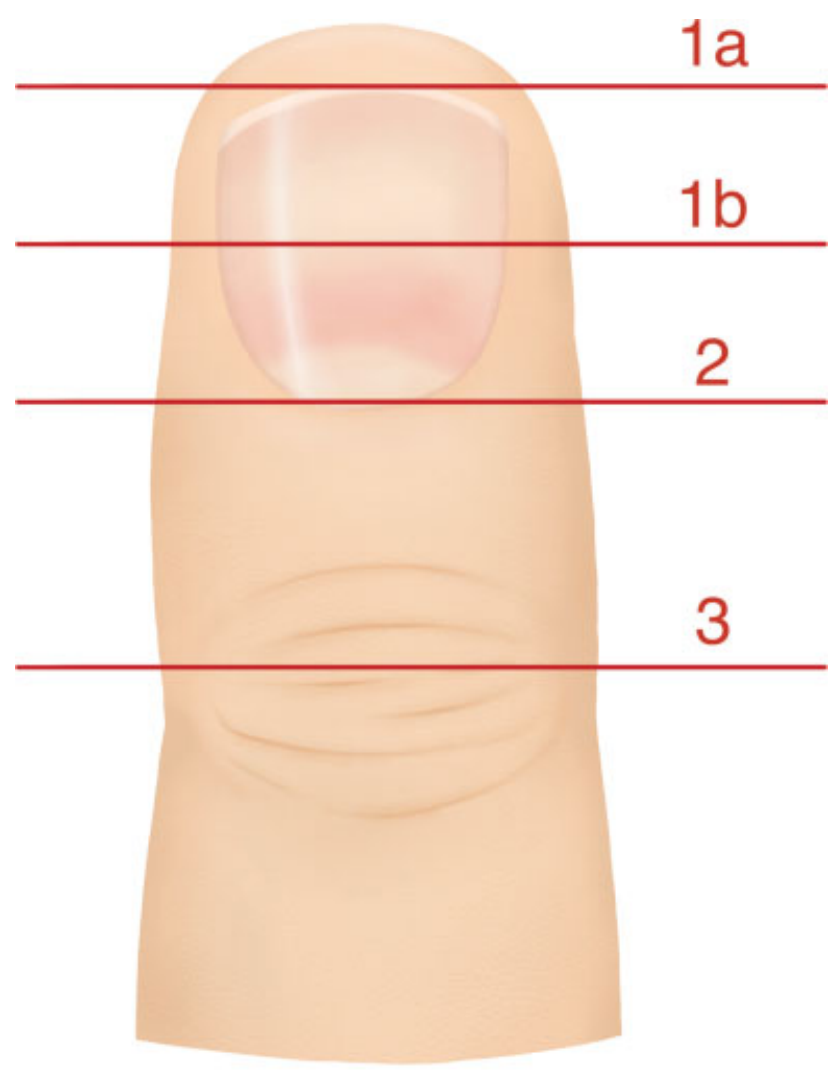

Fig. 6 Clasificación modificada de Ishikawa et al. ${ }^{6}$

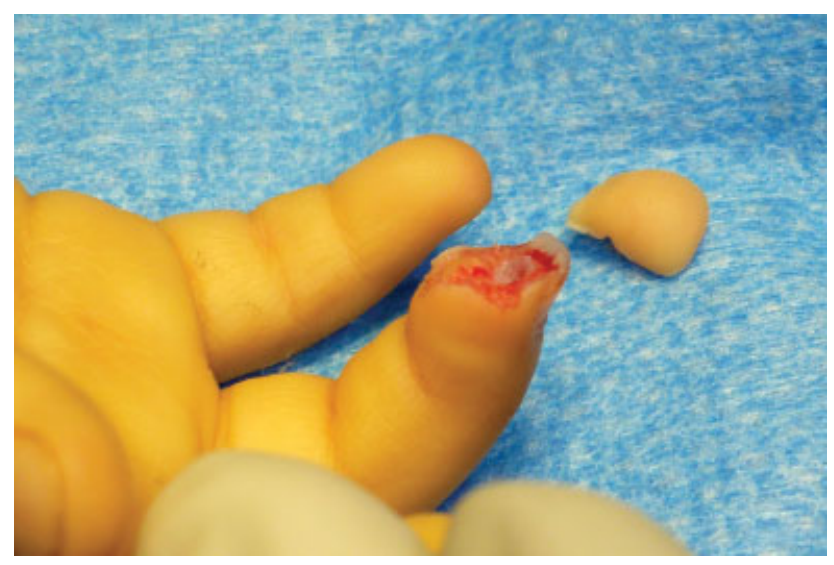

Fig. 7 Amputación de pulpejo del anular derecho, con compromiso óseo.

punta del dedo, sin compromiso óseo, de nivel 1a de acuerdo a la clasificación modificada de Ishikawa et al. ${ }^{6}$ (-Fig. 6). Se realizó aseo, desbridamiento y reconstrucción con injerto compuesto del fragmento amputado, y se dejó con curación semioclusiva por seis semanas. La paciente presentó una recuperación ad integrum de la lesión, sin complicaciones (-Fig. 7, 8, 9, 10, 11).

\section{Caso 2}

Niño de 9 años con atrapamiento de índice derecho en el marco de una puerta, que sufrió una amputación de la punta 


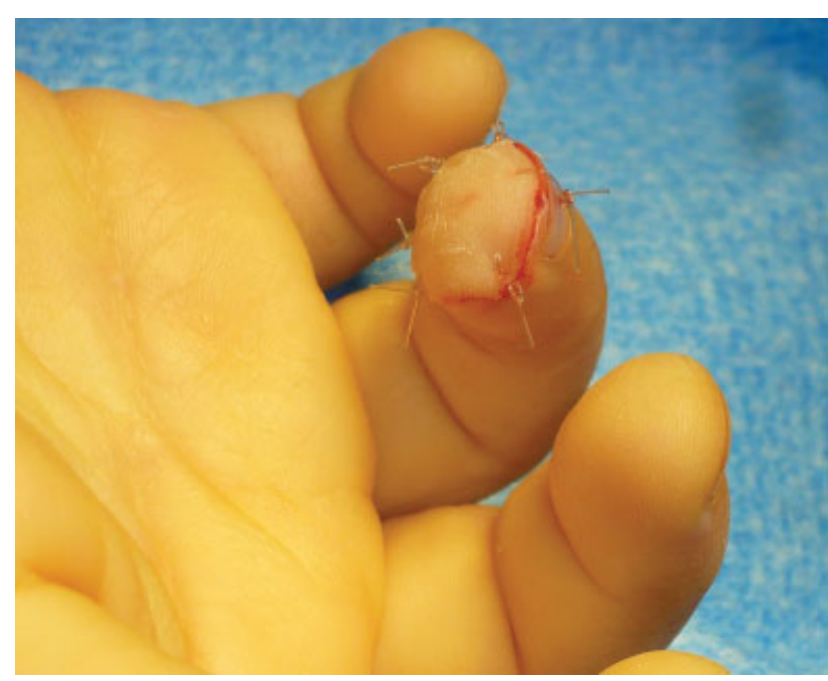

Fig. 8 Reconstrucción de pulpejo con injerto compuesto.

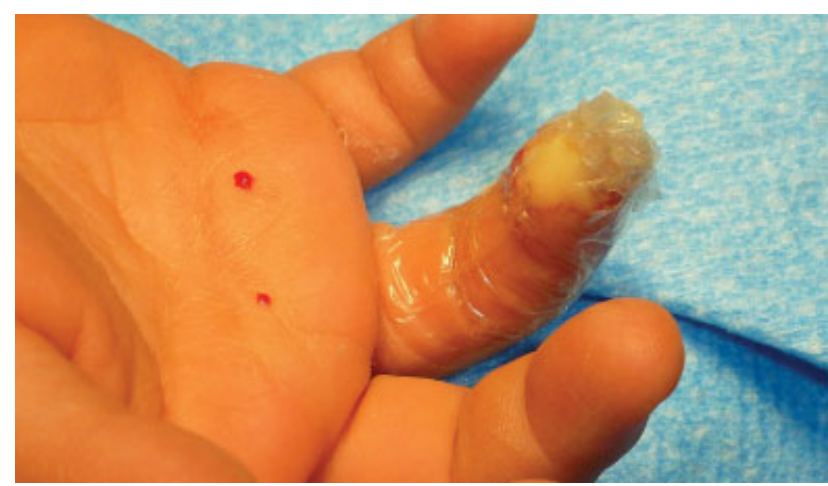

Fig. 9 Colocación de apósito de película transparente.

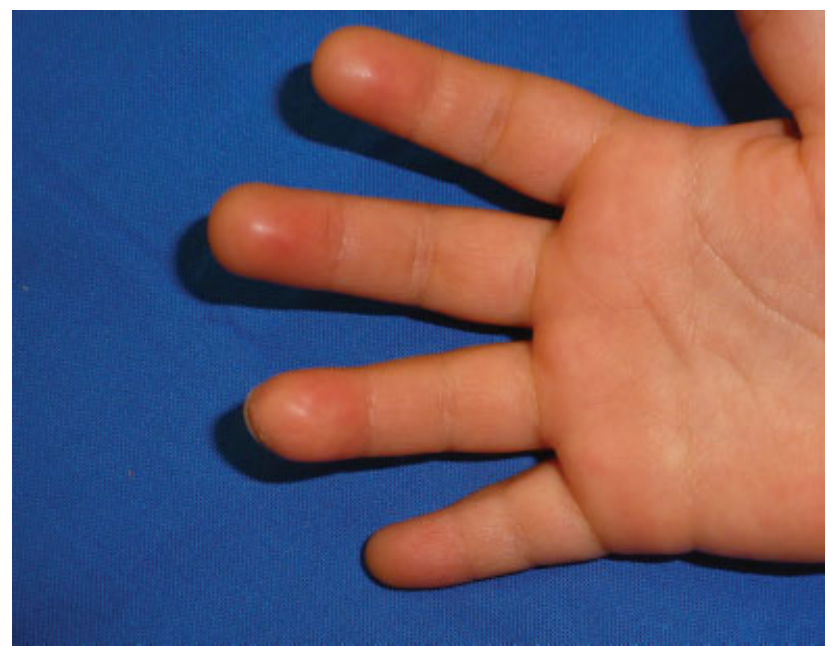

Fig. 10 Control a los trece meses.

del dedo con compromiso de del penacho de la falange distal, de nivel $1 \mathrm{~b}$ en la clasificación modificada de Ishikawa et al. ${ }^{6}$ Se hizo aseo y reconstrucción con injerto compuesto, fijación con aguja del fragmento óseo, y curación semioclusiva (-Fig. 12, 13, 14).

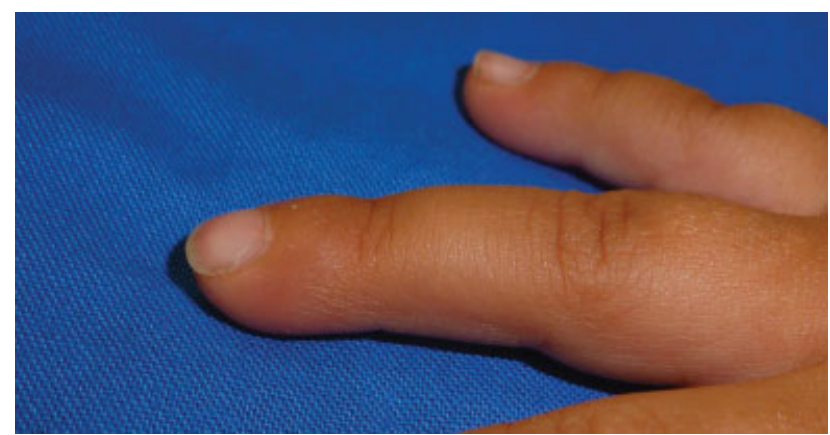

Fig. 11 Pulpejo sin deformación ungeal.

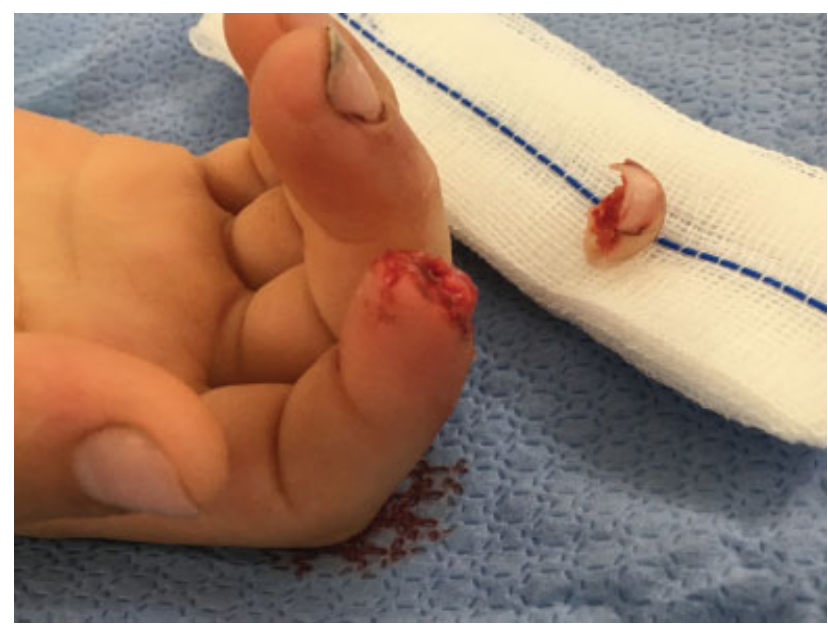

Fig. 12 Amputación de pulpejo del índice derecho.

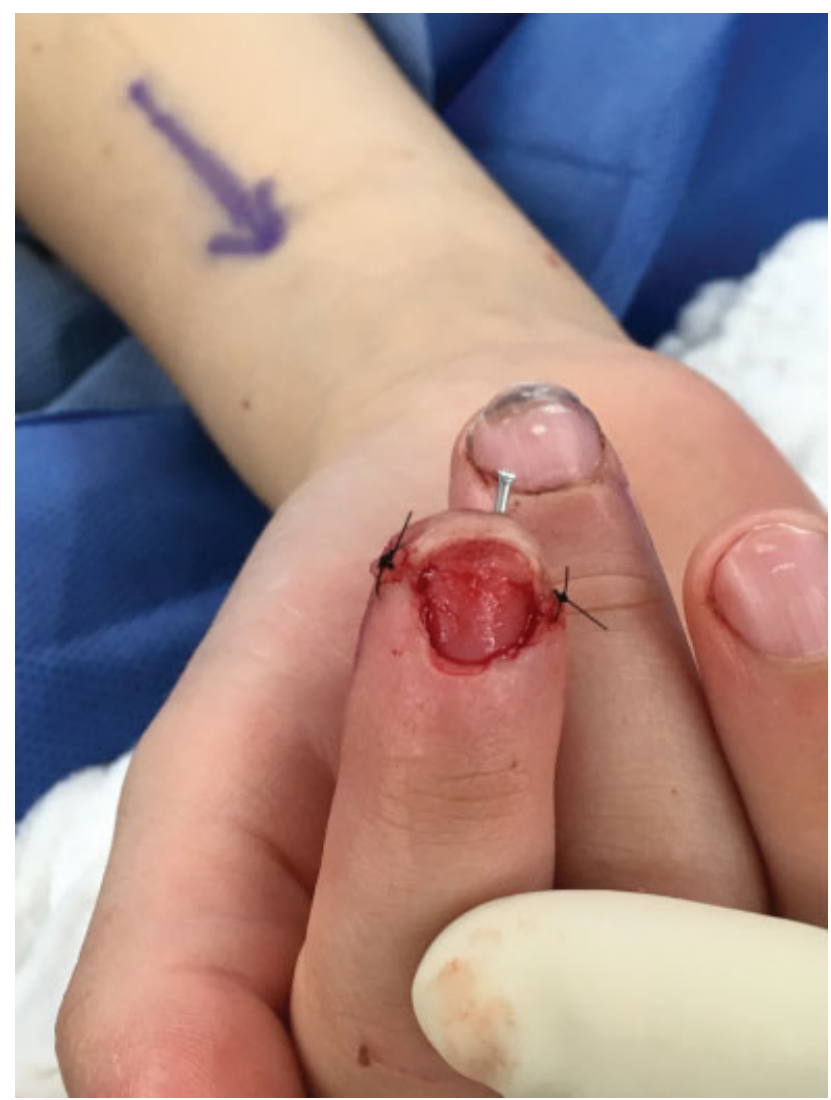

Fig. 13 Reconstrucción con injerto compuesto, con colocación de aguja hipodérmica para fijar fragmento óseo. 


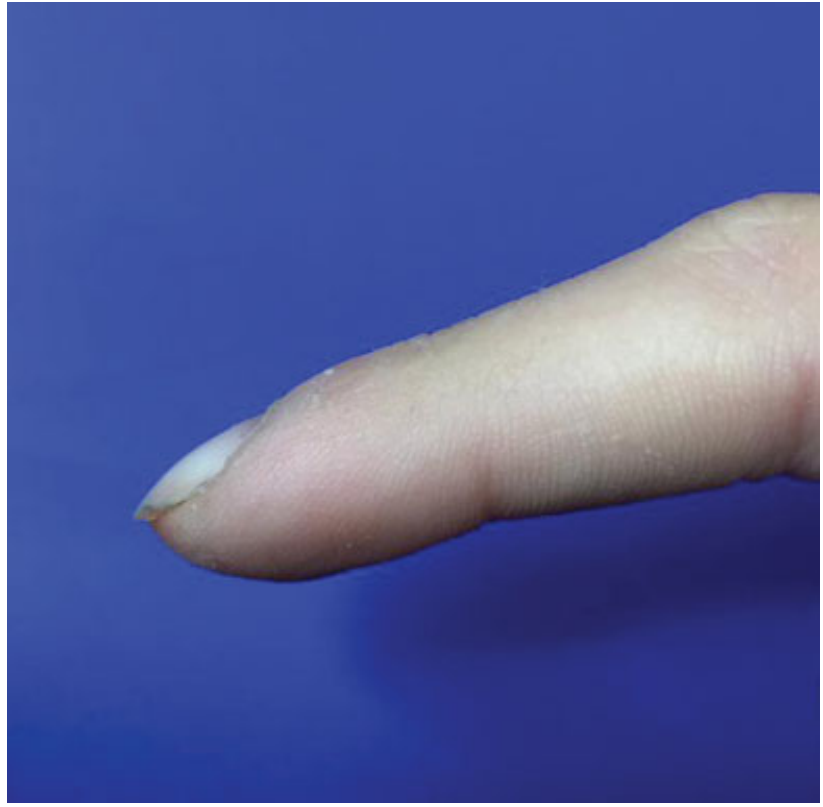

Fig. 14 Aspecto del pulpejo a los doce meses.

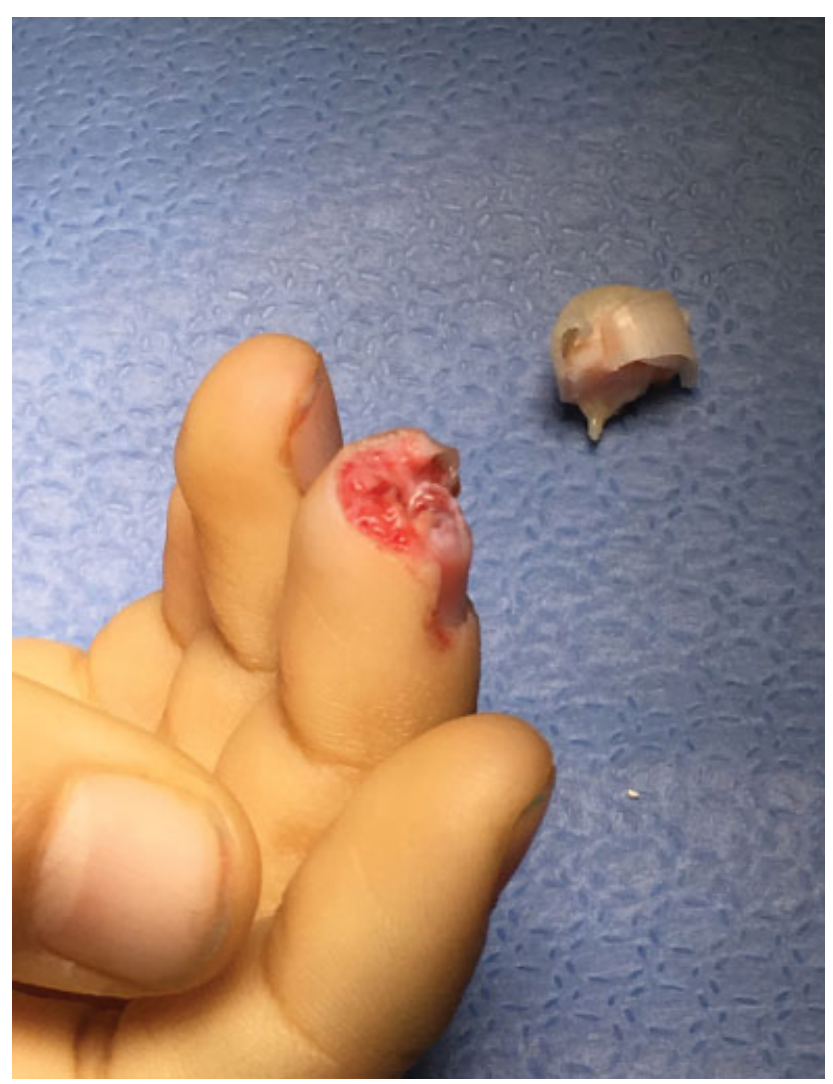

Fig. 15 Amputación de pulpejo del dedo medio derecho.

\section{Caso 3}

Niña de 10 años, quien se atrapó el dedo medio derecho en una puerta, quedando con una amputación de la punta del dedo de nivel 1b en la clasificación modificada de Ishikawa et al. ${ }^{6}$ Se hizo aseo y reconstrucción de dedo con un injerto

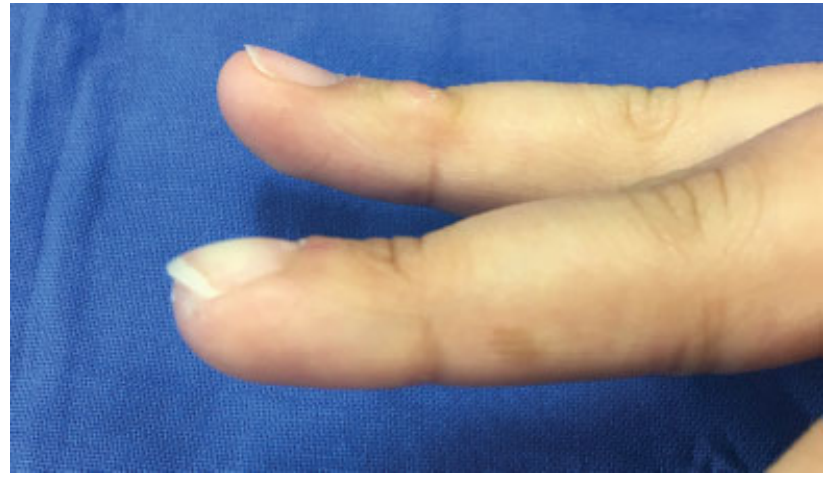

Fig. 16 Aspecto del pulpejo a los doce meses.

compuesto, fijación ósea, y curación semioclusiva. La curación fue retirada a las seis semanas (-Fig. 15, 16).

\section{Discusión}

En la población pediátrica, los intentos por reconstruir la amputación de la punta del dedo no han sido exitosos, y no existe una propuesta definida. Butler et al. ${ }^{5}$ presentaron una serie de 97 casos de reconstrucción de punta de dedos con técnica de reposición de injerto compuesto. Solo el $10 \%$ obtuvo una sobrevida completa del injerto, y 34\%, una sobrevida parcial. Imaizumi et al. ${ }^{7}$ presentaron una serie de 17 reconstrucciones de amputaciones distales de dedos en niños; de estas, 10 fueron con reposición de injerto compuesto, y 5 de ellos sobrevivieron.

Johnson y Giele ${ }^{3}$ presentaron una serie de ocho pacientes en los que hicieron una reconstrucción con injerto de perioniquio (lecho ungeal) y el uso de un colgajo local. Los 4 niños de la serie mostraron una sobrevida completa de la punta del dedo, con un buen resultado estético.

Técnicamente, la reconstrucción de estas amputaciones con el uso de anastomosis con técnicas microquirúrgicas es un desafío, por lo reducido del diámetro de los vasos. Wen et al. $^{8}$ describieron una serie de 21 reimplantes en población pediátrica, con un $95 \%$ de sobrevida de los reimplantes. En mano de equipos entrenados, esta técnica aparece como una buena solución, pero es difícil encontrarlos en nuestra realidad, y también se deben considerar otros aspectos, como el tiempo quirúrgico y los días de hospitalización que se requieren. Hsu et al., ${ }^{4}$ en su serie de 5 reimplantes de punta de dedos, demoraron un promedio de 245 minutos por cirugía, seguidos de 5 días de hospitalización. Imaizumi et al. ${ }^{7}$ relataron 137 minutos de cirugía, con 6,7 días de hospitalización.

Al producirse una herida, en este caso, la amputación distal de un dedo, el proceso de cicatrización clásico que se activa se produce por la contracción de los bordes y una epidermización del tejido cicatricial. ${ }^{9,10}$ La estrategia de la curación semioclusiva ofrece condiciones para que ocurra un proceso diferente de curación. Hoigné et al. ${ }^{1}$ plantearon en su articulo que, frente a una amputación distal de un dedo (en la que no se repone el segmento), la curación semioclusiva permite que se regenere cerca del $90 \%$ del tejido original, y llamaron a esta forma de curación de “curación 
regenerativa", en la que se pueden recrear las condiciones para que ocurra un proceso inflamatorio parecido al que ocurre en etapas embriológicas del desarrollo, ${ }^{11} \mathrm{y}$, en este caso la regeneración se facilitaría a expensas de células madres que se encuentran en el lecho ungeal. ${ }^{12}$ Para que ocurra todo esto, la participación del exudado que se produce tiene un rol crucial. ${ }^{13}$

Es fácil pensar que en este proceso de amputación y reposición del tejido se produce un proceso de muerte celular. Y vemos también que, en el planteamiento de nuestra estrategia de tratamiento, ocurre un proceso de curación ad integrum de la parte amputada.

Con la propuesta de reconstrucción con injerto compuesto y el uso de vendaje semioclusivo, impresiona que, o bien nunca hubo una muerte celular, o bien la hubo, pero, por algún mecanismo, estas células fueron remplazadas, y pensamos que estas repoblaron una zona con estructura tisular conservada. Algo parecido a lo que ocurre con otros procesos biológicos, como la repoblación de tejido celular neural, en la que, frente a la perdida de tejido, los injertos de nervio proveen un exoesquelto tisular que conduce el repoblamiento celular, ${ }^{14}$ o como ocurre con un aloinjerto óseo, en el que el tejido estructural óseo provee el soporte para que se produzca la colonización de células óseas y la consolidación. ${ }^{15,16}$

Sin embargo, proponemos que, al momento de colocar el injerto compuesto, se retire parte del tejido del pulpejo, para disminuir el grosor de este y asegurar una menor área de isquemia.

En relación al nivel de amputación, hay registros que indican que, mientras más proximal es ccccccccla amputación, más posibilidad de necrosis hay ${ }^{3}$ (niveles según la escala modificada de Ishikawa et al. ${ }^{6}$ ), pero nuestra serie de tres pacientes no permite concluir éxito en relación al nivel de amputación.

También se ha descrito la edad como un factor que favorece la sobrevida del injerto compuesto; a menor edad, mejor sobrevida. ${ }^{5}$ Nuestra serie de 3 casos es dispersa, y muestra un paciente de 18 meses, uno de 9 años, y otro de 10 años. Este factor no incidió en el resultado.

Creemos que, para conseguir un buen resultado estético y funcional en la reconstrucción de las amputaciones distales de los dedos en población pediátrica, la conservación del lecho ungeal y del hiponiquio es un hecho de gran importancia. Esto se consigue sólo con la conservación anatómica de las estructuras mencionadas, ya sea por medio de procedimientos microquirúrgicos, o por una reposición del segmento, como un injerto compuesto.

En la serie descrita en este artículo, los niños quedaron con una punta de dedo no dolorosa y con uso de la pinza.

La técnica propuesta oferece una posibilidad más en el tratamiento de estas lesiones en la población pediátrica, sin requerir de técnicas microquirúrgicas. Así, abre la posibilidad de pensar en una técnica sencilla y fácil de reproducir.

Este procedimiento necesita más casos para su validación.

Conflicto de Intereses

Los autores declaran que no hay conflicto de intereses.

\section{Bibliografía}

1 Hoigné D, Hug U, Schürch M, Meoli M, von Wartburg U. Semiocclusive dressing for the treatment of fingertip amputations with exposed bone: quantity and quality of soft-tissue regeneration. J Hand Surg Eur Vol 2014;39(05):505-509

2 Adani R, Marcoccio I, Tarallo L. Treatment of fingertips amputation using the Hirase technique. Hand Surg 2003;8(02): 257-264

3 Johnson D, Giele H. Restoration of the nail apparatus following fingertip amputation by perionychial grafts supported by local flaps, in children and adults. J Plast Reconstr Aesthet Surg 2011;64 (06):776-781

4 Hsu CC, Lin YT, Moran SL, Lin CH, Wei FC, Lin CH. Arterial and venous revascularization with bifurcation of a single central artery: a reliable strategy for Tamai Zone I replantation. Plast Reconstr Surg 2010;126(06):2043-2051

5 Butler DP, Murugesan L, Ruston J, Woollard AC, Jemec B. The outcomes of digital tip amputation replacement as a composite graft in a paediatric population. J Hand Surg Eur Vol 2016;41(02): 164-170

6 Ishikawa K, Ogawa Y, Soeda H, Yoshida Y. A new classification of the amputation levelfor the distal part of the finger. J Jpn Soc Microsurg 1990;3:54-62

7 Imaizumi A, Ishida K, Arashiro K, Nishizeki O. Validity of exploration for suitable vessels for replantation in the distal fingertip amputation in early childhood: replantation or composite graft. J Plast Surg Hand Surg 2013;47(04):258-262

8 Wen G, Xu J, Chai YM. Fingertip replantation with palmar venous anatomoses in children. Ann Plast Surg 2017;78(06):692-696

9 Goitz RJ, Westkaemper JG, Tomaino MM, Sotereanos DG. Softtissue defects of the digits. Coverage considerations. Hand Clin 1997;13(02):189-205

10 Martin C, González del Pino J. Controversies in the treatment of fingertip amputations. Conservative versus surgical reconstruction. Clin Orthop Relat Res 1998;(353):63-73

11 Roshan A, Grant I. Lessons for adult fingertip regeneration: glimpses from basic research. J Hand Surg Am 2012;37(06): $1287-1290$

12 Choi Y, Cox C, Lally K, Li Y. The strategy and method in modulating finger regeneration. Regen Med 2014;9(02):231-242

13 Mennen U, Wiese A. Fingertip injuries management with semiocclusive dressing. J Hand Surg [Br] 1993;18(04):416-422

14 Williams LR, Longo FM, Powell HC, Lundborg G, Varon S. Spatialtemporal progress of peripheral nerve regeneration within a silicone chamber: parameters for a bioassay. J Comp Neurol 1983;218(04):460-470

15 Gil J, Garrido R, Gil R, Melgosa M. Materiales para la reparación y sustitución ósea. Factores de crecimiento y terapia genética en Cirugía Ortopédica y Traumatología. Mapfre Medicina 2003; 14:51-65

16 Calvo R, Figueroa D, Díaz-Ledezma C, Vaisman A, Figueroa F. Aloinjertos óseos y la función del banco de huesos. Rev Med Chil 2011;139(05):660-666 